\title{
FIELD AND CIRCUIT MODELS FOR THE WOUND FOIL INDUCTOR
}

\author{
P. N. MURGATROYD \\ Department of Electronic \& Electrical Engineering, Loughborough University of Technology, \\ Loughborough, Leicestershire, England.
}

(Received May 20, 1976)

\begin{abstract}
The Wound Foil Inductor is an important example of inductive components with appreciable internal capacitance. It is examined from three viewpoints - electromagnetic field theory, distributed-parameter (or transmission-line), and lumped equivalent circuit. The analyses are compared, particularly in terms of phase gradients within a component, and the relationship between a two-dimensional field analysis and the now established lumped model is derived.
\end{abstract}

\section{INTRODUCTION}

The Wound Foil Inductor (WFI) is mechanically a very simple component. A single strip of metal foil, usually aluminium, is wound with a slightly wider strip of polymer film or other insulating layer onto a suitable former. The basic construction is shown in Figure 1. Interest in such coils has arisen in two ways Firstly, for power applications it was recognised that aluminium is lighter and in the long run potentially cheaper than copper in completed components, foil can be wound with a higher space-factor than round wire, and the construction has proven superior heat dissipation properties because all conductors provide their own heat paths to the outside. Secondly, it was

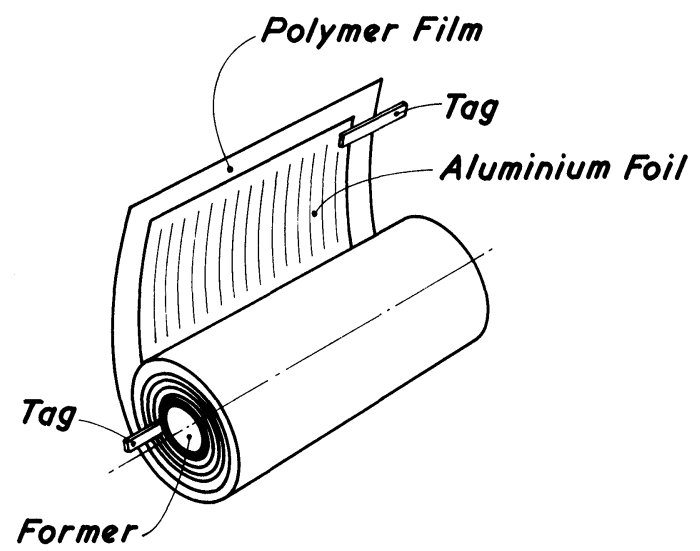

Construction of Wound Foil Inductor. FIGURE 1 known that certain coiled constructions for capacitors produced components with self-resonant properties at high frequencies, and it was thought that coiled constructions of one foil, as opposed to the two foils of a capacitor, might offer a pathway to better understanding of such effects.

The WFI in fact presented a formidable problem on its own. Figure 2 shows how the impedance of a simple 1000 turn foil inductor varies in the radio frequency range. The measurement is made by connecting the inductor in series with a white noise source and a sweeping-filter spectrum analyser, so that in the display a minimum of current represents a maximum of impedance, and vice-versa. These impedance variations can be explained, and the peak

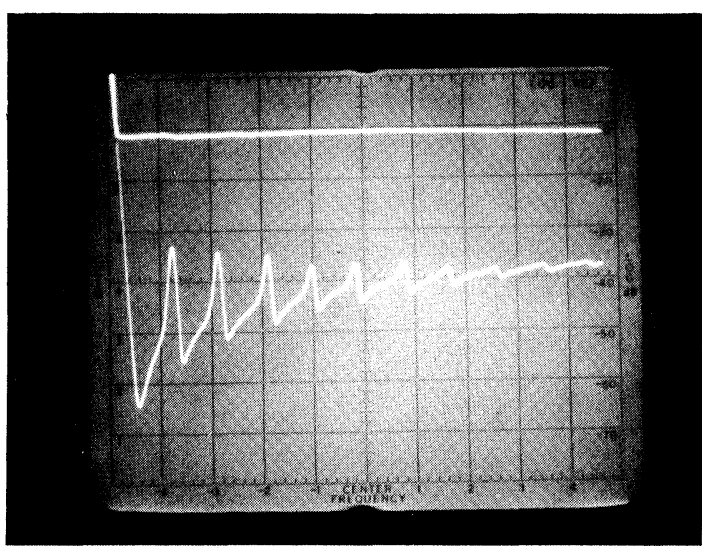

FIGURE 2 Spectral display of impedance of a 1000-turn wound foil inductor. Abscissa: $1 \mathrm{MHz}$ per square, range 0 to $10 \mathrm{MHz}$; Ordinate: $10 \mathrm{~dB}$ attenuation per square, zero at top. 
frequencies predicted from first principles with useful accuracy 1,2 in terms of a lumped equivalent circuit, such as Figure $3 b$.

It is interesting to note, however, that when confronted with results such as Figure 2, the most common reaction of engineers has been 'it must behave like a transmission line.' For reasons which are detailed in $\S 5$ this view has usually been rejected in studies at Loughborough and it contributed little to the workable equivalent circuit model ${ }^{1}$. However the WFI can in fact be regarded as a problem requiring field analysis for full solution, or (as $\$ 4$ of this paper demonstrates) in a simplified form as a problem amenable to distributed-parameter analysis redolent of elementary transmission-line theory. It is the aim of this paper to relate these viewpoints, and to suggest how, having described the WFI, other coiled devices might be analysed similarly.

\section{LUMPED CIRCUIT MODEL}

Each turn of the WFI is regarded as a pure inductance in series with a pure resistance. The capacitance between each turn and its neighbour on either side is represented by a pure capacitance connected between the mid-points. Figure 3a shows this arrangement, and it is understood that in practice all lumped values will depend on position in the WFI, with magnitudes increasing from the inner to the outer windings. Each turn is mutually coupled to all other turns; it is not possible to show all such couplings in the diagram.

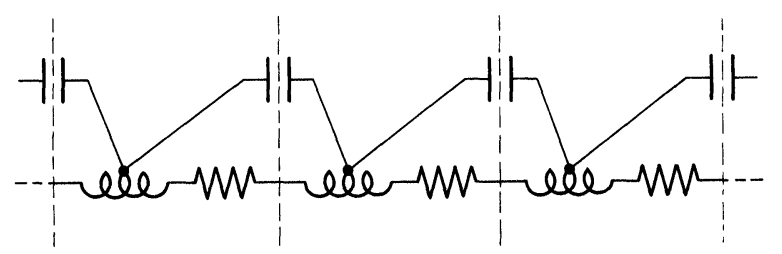

(a)

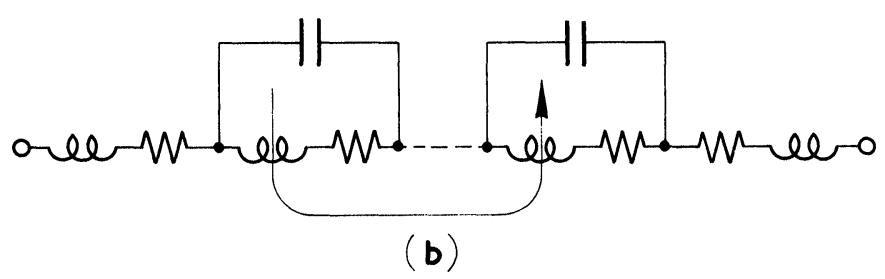

\section{Development of Lumped Circuit Model}

(N.B. All Inductors ore Mutually Coupled)

FIGURE 3
To reach the working equivalent circuit the diagram is re-arranged as in Figure 3b, and (provided the total number of turns is large) half-turn elements at each end of the coil are simply neglected. All lumped values in this circuit can be calculated from first principles. However since all values are different the calculation can be cumbersome, even on a large computer. One way of overcoming this problem is to group together groups of, say, 10 adjacent turns $\mathbf{1}, \mathbf{2}$ and analyse a lumped-lumped circuit, still represented by a diagram like Figure $3 \mathrm{~b}$ but with one tenth of the number of sections and one hundredth of the number of couplings.

\section{FIELD ANALYSIS}

As to all electromagnetic systems, Maxwell's Equations apply to Wound Foil Inductors, and if all else fails solution for the fields is the method of last resort. In principle one must be able to find the magnetic and electric fields, and the current density, everywhere, even including their variations across the widths and within the thicknesses of the metal foils and dielectric films. In a spiral geometry, analytical answers cannot be expected, and a numerical grid solution in three dimensions might easily demand hundreds of unknowns to represent each foil turn adequately. Thus major approximations seem inevitable.

Firstly, therefore, we shall neglect field variations within the foil and film thicknesses. In the dielectric this means assuming that the foil is locally flat, the radius of curvature being much larger than the foil thickness. In the metal it means uniform current density across the thickness, or nominal skin depth and radius of curvature much larger than foil thickness.

To neglect field variations across the foil width is a more serious step. It is clear, for example, that when a single foil turn carries a steady current both the axial and radial magnetic fields will vary with axial distance. With alternating current this will lead, it is believed ${ }^{3}$, to a non-uniform distribution of current across the foil width, and to an effective a.c. resistance appreciably greater than the d.c. resistance. In terms of the fields the situation is simply described. At every point in the WFI there is an inductive effect, expressible as an induced electric field, given locally by the rate of change of $\mathbf{A}$, the magnetic vector potential ${ }^{4}$. However the value of $\mathbf{A}$ is determined by the current density distribution everywhere in the coil. Thus the current density at one point is affected by the current density at all other points; ultimately 
the whole coil must be regarded as a self-consistent field problem. Notwithstanding this fundamental (and in practice possibly insuperable) difficulty, some progress can be made by neglecting variations in the width, and then the problem may be treated as one in distributed-parameter analysis.

\section{DISTRIBUTED PARAMETER ANALYSIS}

The cross-section of the metal foil, with negligible thickness and all spaces filled by dielectric, is shown in Figure 4 and described by the polar equation

$$
r=a+l \frac{\theta}{2 \pi}, \quad 0<\theta<2 N \pi
$$

Here $a$ is the core radius, and $l$ is the spiral increment per turn, related to the outer radius $b$ and number of turns $N$ by

$$
b=a+N l
$$

We now neglect all field variations across the foil width, and hence any frequency-dependence of resistance. We define a resistance $\Lambda$ per unit length measured along the foil, so the resistance between neighbouring points given by angles $\theta$ and $\theta+\mathrm{d} \theta$ will be $\Lambda r \mathrm{~d} \theta$. Similarly we define a capacitance $\Gamma$ per unit length between adjacent foils. The local equations for the voltage and current profiles $V(\theta)$ and $I(\theta)$ are derived from Figures $5 \mathrm{a}$ and $5 \mathrm{~b}$. The sign convention is that $V(\theta)$ increases from the centre outwards and $I(\theta)$ is positive when flowing inwards.

In Figure 5a the current in a small section of foil is given by

$$
I \Lambda r \mathrm{~d} \theta=\frac{\partial V}{\partial \theta} \cdot \mathrm{d} \theta-\frac{\partial A_{\theta}}{\partial t} \cdot r \cdot \mathrm{d} \theta
$$

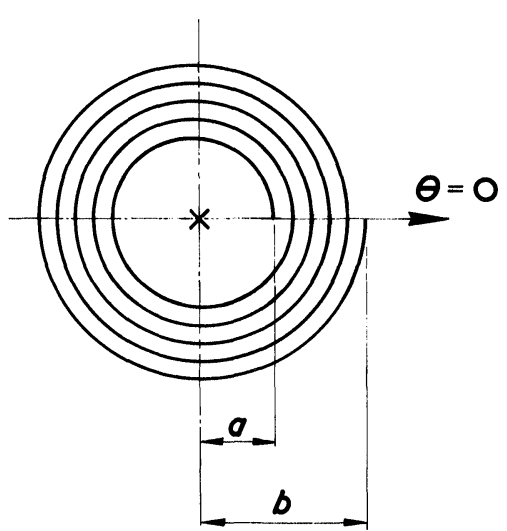

Spiral Geometry of Coil Section.

FIGURE 4

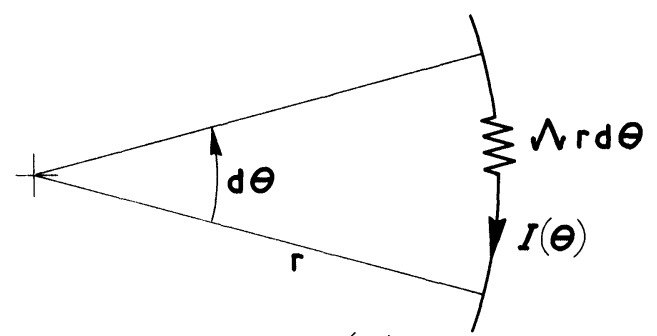

(a)

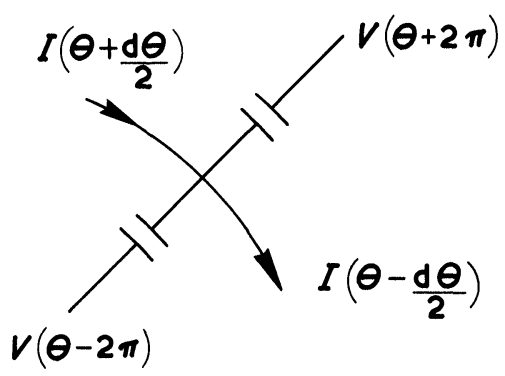

(b)

\section{Coil Elements for Distributed Parameter Analysis}

FIGURE 5

where $A_{\theta}$ is the local circulating component of the magnetic vector potential. In the limit of small $\mathrm{d} \theta$ we have

$$
I \Lambda=\frac{1}{r} \cdot \frac{\partial V}{\partial \theta}-\frac{\partial A_{\theta}}{\partial t}
$$

For the steady state this equation may be integrated for $\theta$ ranging from zero to $2 N \pi$ to give the d.c. resistance

$$
R_{\text {d.c. }}=\frac{V}{I}=\pi N(a+b) \Lambda
$$

In Figure $5 \mathrm{~b}$ we must conserve current at the junction, therefore

$$
\begin{aligned}
0= & -I\left(\theta-\frac{\mathrm{d} \theta}{2}\right)+I\left(\theta+\frac{\mathrm{d} \theta}{2}\right) \\
& +\Gamma \cdot\left(r+\frac{l}{2}\right) \mathrm{d} \theta \cdot \frac{\partial}{\partial t}(V(\theta+2 \pi-V(\theta)) \\
& -\Gamma \cdot\left(r-\frac{l}{2}\right) \mathrm{d} \theta \cdot \frac{\partial}{\partial t}(V(\theta)-V(\theta-2 \pi))
\end{aligned}
$$


At this stage we neglect the small difference between the two capacitances due to stacking of turns

$$
\begin{gathered}
\left(\frac{l}{2} \ll r\right) \text { and obtain } \\
\frac{1}{r} \cdot \frac{\partial I}{\partial \theta}=\Gamma \cdot \frac{\partial}{\partial t}[2 V(\theta)-V(\theta+2 \pi) \\
-V(\theta-2 \pi)]
\end{gathered}
$$

In the steady state, integration of this equation yields the expected uniform current.

Now we attempt to integrate the field equations (4.4) and (4.6), each for one turn of the inductor. For Eq. (4.4) we write

$$
\begin{aligned}
\int_{\theta}^{\theta+2 \pi} I \cdot \Lambda \cdot \mathrm{d} \theta= & \int_{\theta}^{\theta+2 \pi} \frac{1}{r} \cdot \frac{\partial V}{\partial \theta} \cdot \mathrm{d} \theta \\
& -\frac{\partial}{\partial t} \int_{\theta}^{\theta+2 \pi} A_{\theta} \cdot \mathrm{d} \theta
\end{aligned}
$$

To achieve this integration we must let $I, r$ and $A_{\theta}$ each take a mean value for the turn, denoted by the suffix $m$, and these values are related to the chosen range of integration characterised by the common lower limit $\theta$. Then we obtain

$$
2 \pi I_{m} \Lambda=\frac{2 \pi}{r_{m}}(\Delta V)_{m}-\frac{\partial}{\partial t}\left(2 \pi A_{\theta, m}\right)
$$

Now we note that the resistance of the turn is given by $R_{m}=\Lambda r_{m}$, and that the magnetic flux enclosed by the turn is equal to the line-integral of the vector potential around the turn, i.e. $\phi_{m}=2 \pi r_{m} A_{\theta, m}$, and so

$$
I_{m} R_{m}=(\Delta V)_{m}-\frac{\partial}{\partial t}\left(\Phi_{m}\right)
$$

The same integration procedure may be applied to Eq. (4.6) and we obtain the result

$$
\begin{aligned}
(\Delta)_{m}= & -C_{m}^{+} \frac{\partial}{\partial t}(V(\theta+2 \pi)-V(\theta)) \\
& +C_{m}^{-} \frac{\partial}{\partial t}(V(\theta)-V(\theta-2 \pi))
\end{aligned}
$$

where the capacitances are given by $C_{m}^{ \pm}=2 \pi(r \pm l / 2) \Gamma$

At this stage we must be flexible in interpreting the suffix $m$, for in Eq. (4.10) the term $(\Delta V)_{m}$ denotes the voltage difference between the ends of the turn, but this will scarcely differ from the voltage difference between the midpoint of the chosen turn and that of an adjacent turn. A similar interpretation is applied to the currents. We also now assume harmonic excitation, and for equations (4.10) and (4.11) we have

$$
\begin{aligned}
I_{m} R_{m}=V_{m} & \cdots V_{m-1}-j \omega \Phi_{m} \\
I_{m+1}-I_{m}= & j \omega C_{m}\left(V_{m}-V_{m+1}\right) \\
& -j \omega C_{m+1}\left(V_{m+1}-V_{m}\right)
\end{aligned}
$$

From the last two equations the voltages may be eliminated to give

$$
\begin{aligned}
I_{m+1}-I_{m}= & j \omega C_{m}\left(I_{m} R_{m}+j \omega \Phi_{m}\right) \\
& -j \omega C_{m+1}\left(I_{m+1} R_{m+1}+j \omega \Phi_{m+1}\right)
\end{aligned}
$$

and the terms referring to adjacent turns may be separated to give

$$
\begin{aligned}
T & =I_{m+1}+j \omega C_{m+1}\left(I_{m+1} R_{m+1}+j \omega \Phi_{m+1}\right) \\
& =I_{m}+j \omega C_{m}\left(I_{m} R_{m}+j \omega \Phi_{m}\right)
\end{aligned}
$$

where $T$ is evidently some invariant relating all turns of the coil to each other.

The meaning of $T$ is apparent when we consider the circuit of Figure 6, in which the mesh equation for the $u^{\text {th }}$ loop is

$$
I_{u} R_{u}+j \omega X_{u}+\frac{I_{u}-I_{0}}{j \omega C_{u}}=0
$$

Here $X_{u}$ is a composite inductance including both the self-inductance of the $u$ th loop and mutual couplings from all other loops. We identify $X_{u}$ with the reactive flux term $\Phi_{m}$ in Eq. (4.15). We identify $R_{u}$ with the turn resistance $R_{m}$. Finally we identify the external current $I_{0}$ with the invariant $T$. Thus we have shown

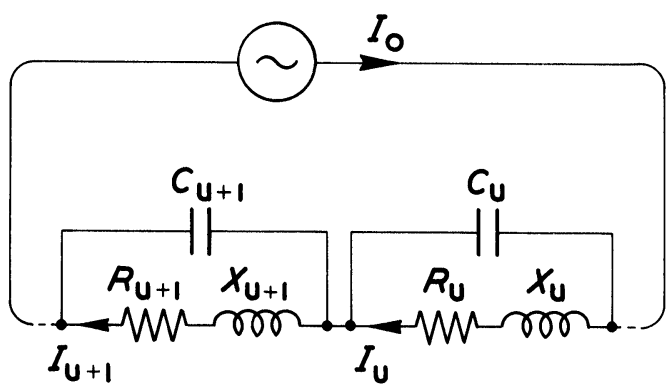

Lumped Circuit derived from

2-D Field Theory.

FIGURE 6 
that, excepting difficulties with the innermost and outermost half-turns (which are neglected in both analyses) the distributed parameter method leads to essentially the coupled equivalent circuit on which the successful quantitative theories have been based.

\section{DISCUSSION}

The observed behaviour of the WFI, in particular the multiple resonances which superficially appear to be (but are not) uniformly spaced in frequency, has suggested a transmission-line model might be appropriate. It might perhaps now be argued that this is the case. The distributed-parameter or twodimensional field, method leads to equations for the rate of change of voltage and current with position inside the system, and thus recalls the elementary equations of a transmission line. There are however important differences. The transmission-line has four terminals; the WFI has two. In transmission-line theory the leakage capacitance is local, between the conductors; in the WFI the capacitance is non-local, for it is between different parts of the same conductor, and those different parts are not even at constant separation along the conductor. Finally in transmission-line theory the inductive effect is local, and a signal must effectively pass along the line to reach the other end. In the WFI there is appreciable mutual inductance between all parts, so if we are to conceive of a signalling time between the ends it is the time taken in travelling from inner to outer across, not along, the conductive windings.

The levels of analysis outlined in this paper are perhaps best discussed in terms of phase. In the simplest analysis of an inductive coil it is implicitly assumed, though rarely stated, that the current in all parts of the coil is in the same phase, exactly. The simplest description of self-resonance represents a coil by inductance and capacitance in parallel, just one of each, and the internal phase distribution is not considered. If the coil is regarded as a number of subcoils, as in the lumped--lumped calculation ${ }^{1,2}$ then it is understood that there may be a phase change from one sub-coil to the next, but none within each sub-coil. Such a model is limited to describing low orders of self-resonance, to an order (say) no greater than half the number of sub-coils. The lumped model of $\$ 2$ permits finer description: here there may be a phase change from one turn to the next, but it is discontinuous, with no phase gradient within a turn. The two-dimensional distributed model of $\S 4$, in the general formulation, admits a phase gradient every- where which could in principle be calculated. The integration of this model, formally deriving the lumped circuit from the field equations, hinged on approximations over an individual turn which effectively averaged the internal phase gradient and made necessary a phase jump from one turn to the next, wherever the division was taken to be. Finally, a field analysis would show a phase gradient across the foil width (as in the classical skin effect) as well as around the foil turns.

It is perhaps paradoxical that capacitive effects in a foil coil are more easily understood than in a wire coil, for in the wire coil all the inter-turn capacitances are much smaller. They are however organised in a much more complicated fashion. In the foil coil there is a large capacitance between each turn and its immediate neighbours, and though there will be edgecapacitances between second and more distant neighbours they are orders of magnitude smaller. In a wire coil each turn may have eight near neighbours, and any node-diagram corresponding to Figure $5 b$ would show at least ten current-paths, and possibly many more, for second-neighbour capacitances may not be negligible and are certainly more numerous. Excepting this complication, however, the basic theory of $\S 4$ is formally applicable to a wire coil: the equation (4.4) is unchanged, and Eq. (4.6) acquires more terms, possibly with several different values for the distributed capacitance $\Gamma$. Although as yet no formal work has been done, it appears that transformers and all related foil or wire coil devices could also be examined in the same way.

\section{CONCLUSION}

A general theory of wound components has been presented, and applied in detail to Wound Foil Inductors. The different viewpoints of field theory, distributed-parameter analysis and lumped-parameter analysis have been compared and related through their descriptions of phase-change within a device. For the WFI it is shown that the latter two viewpoints lead formally to the same equivalent circuit when the voltage and current profiles are suitably averaged.

\section{ACKNOWLEDGEMENTS}

The problem of resonance in foil coils was first brought to the author's attention by Professor D. S. Campbell. The theories have been clarified in discussions with Dr. R. Reeves, Dr. A. R. Morley, Mr. R. J. Kemp and Mr. N. J. Walker. 


\section{REFERENCES}

1. R. J. Kemp, P. N. Murgatroyd and N. J. Walker, 'Self resonance in foil inductors', Electronics Letters, 11, 337 (1975).
2. N. J. Walker, 'Multiple resonance in foil wound inductors', M.Sc. Thesis, Loughborough University (1975).

3. R. Reeves, Unpublished (1974).

4. R. Stratton, Electromagnetic Field Theory, (McGrawHill, New York, 1941), pp. 28-32. 

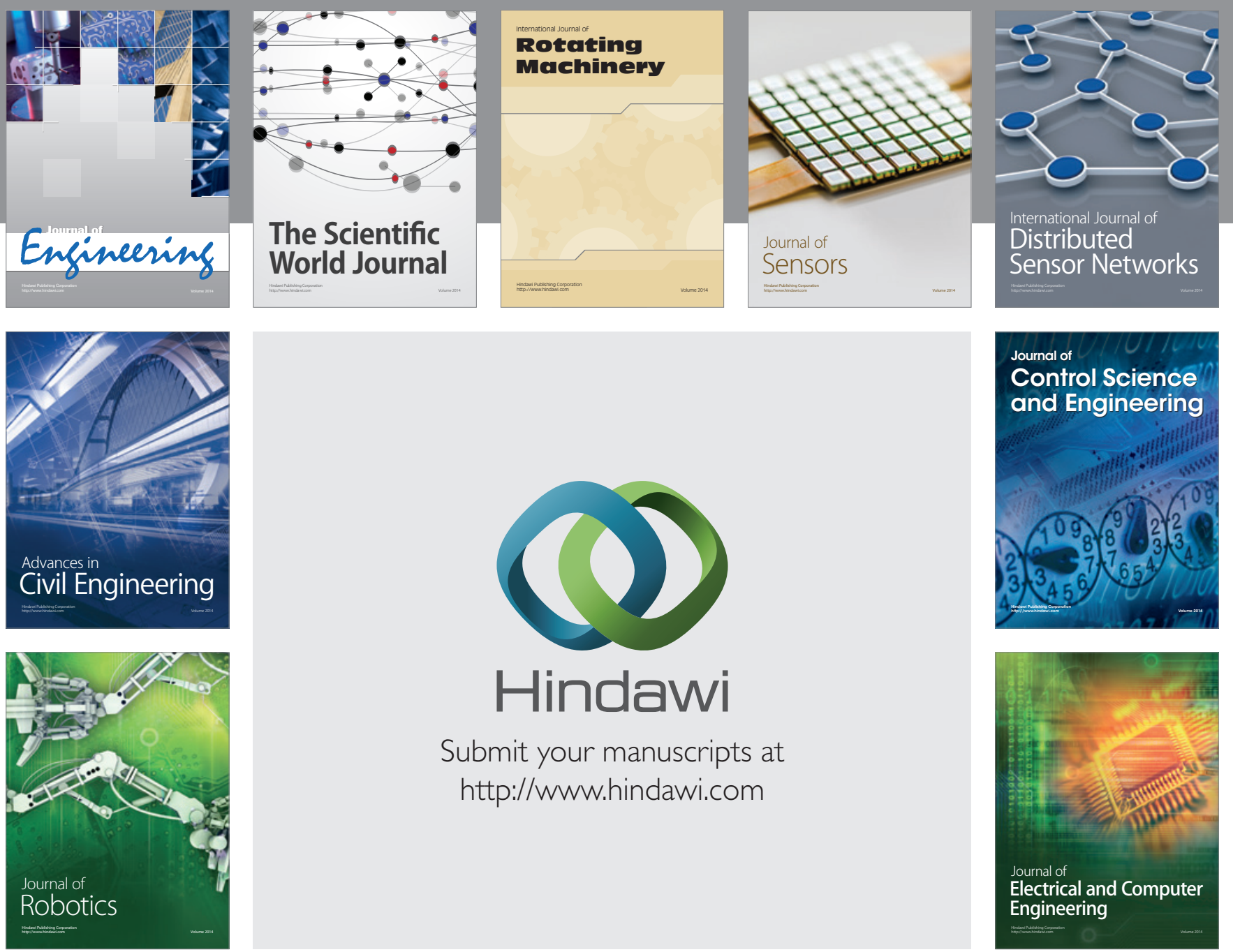

Submit your manuscripts at

http://www.hindawi.com
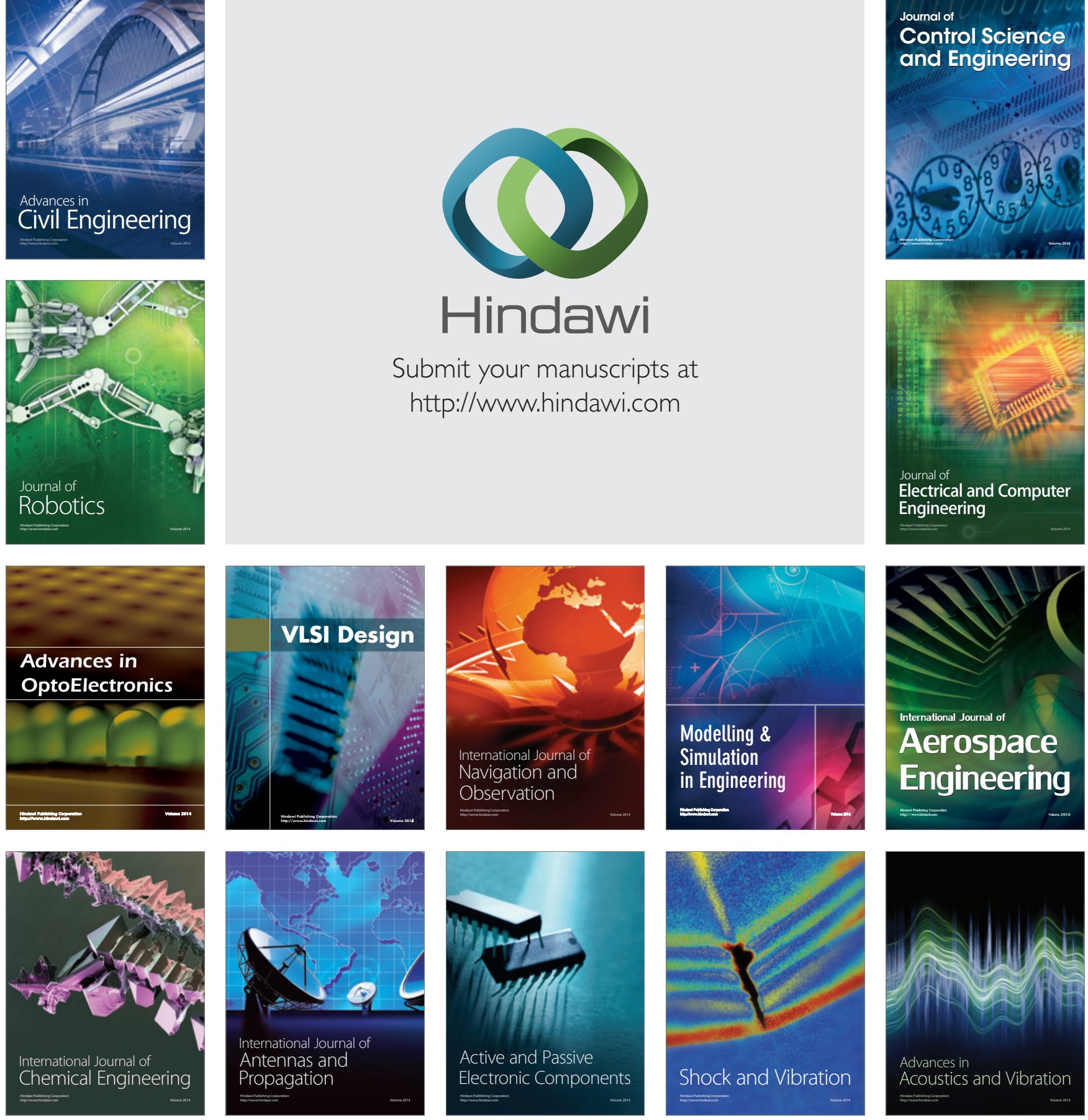